\title{
Rapid Anxiety Reduction Tools for Nursing Staff, Faculty and Students
}

\author{
Anne M Devney* \\ Penn State University Mont Alto campus, USA \\ *Corresponding author: Anne M Devney, Penn State University Mont Alto campus, USA
}

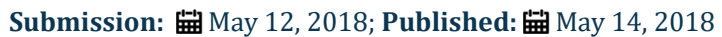

\section{Learning Objectives}

A. Review use of mindfulness as a stress reduction technique.

B. Review rapid anxiety reduction techniques that faculty members can use.

\section{Review those strategies.}

The stressors present for nursing staff, faculty and students are factors that can be addressed with simple non-invasive strategies. Such techniques can be used in the classroom, simulation lab, and the clinical area. Encouragement of simple mindfulness techniques can assist with stress and anxiety reduction. Knowing that elements of time availability play a major role in any setting, one might consider strategies that are appropriate to use in the context of the situation.

In addition to selection of stress reduction techniques, the nurse and nurse educator might consider how use of these elements may help foster improved critical thinking. The National League for Nursing's Guide for Teaching Thinking calls for exploring context, content and course of going forward. Reflection is a critical component of assessing the relevance of content and how to use the information to go forward. Using mindfulness can provide the nurse, nursing student, and nurse educator with means for enhancing critical thinking.

Mindfulness is defined as awareness of the present moment, being nonjudgmental, simply having a sense of who and where you are. Dr. Jon Kabat-Zinn, University of Massachusetts, developed a Mindfulness-Based Stress Reduction (MBSR) program several years ago. Program elements assist the individual to foster awareness of the present moment and uses techniques such as breathing, meditation, yoga, and a body-scan awareness exercise. Mindfulness increases awareness of one's 5 senses, noticing landscapes of your mind without holding on or pushing out what one is feeling [1].

Research in use of Mindfulness-Based Stress Reduction is underway studying medical and nursing students with reports of participants feeling less anxiety and more calmness. Practitioners of mindfulness can use autogenic techniques incorporating progressive relaxation with meditation and guided imagery.

Psychological issues in the clinical and academic setting are well known and include stressors that may be too overwhelming, reducing students' and clinicians' performance. Physical signs such as tremors, agitation, poor concentration, problem-solving abilities, and lack of attention can result in poor task performance and a lack of confidence. Coping skills may be overwhelmed.

Techniques that can be used range from immediate to short term to longer term time frames. For example, if you have 1 minute, take a nice deep breath and feel the air moving in and out of your chest. Feel your body's sensations, the air about you, listen to the sound of your breathing. If you have 2 minutes, breathe in to a count of 10 , breathe out to a count of 10 . Feel your chest rise \& fall. As you exhale, feel your muscles relax down to your feet. If you have 3 or more minutes, tighten up your forehead to a count of 3 . Relax to a count of 3 . Tighten up your shoulders to a count of 3 and relax to a count of 3. Work progressively down the body with muscle groups. Feel those muscles tighten and relax [2].

Of course, the techniques listed above are simply suggestions especially with noted time constraints. Meditation and yoga techniques used over longer periods of time are very useful and have been proven to be very effective. Success of techniques vary with each individual; many people are hesitant to try some mindfulness techniques and feel as though they will not work. However, the time used to focus one's attention to the present moment can be used to develop awareness of the context of the situation and encourage reflection of the meaning within that context. Such reflection itself may provide that individual with a pause that refreshes the thinking of the moment, possibly reducing anxiety and associated stressors.

\section{References}

1. Forneris SG, Fey M (2018) Critical conversations the NLN guide for teaching thinking. Wolters Kluwer, Philadelphia, USA.

2. Kabat Zinn J (2018) https://www.mindful.org/jon-kabat-zinn-definingmindfulness/ 
Creative Commons Attribution 4.0 International License

For possible submissions Click Here

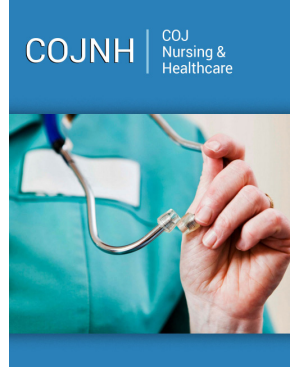

\section{COJ Nursing \& Healthcare}

\section{Benefits of Publishing with us}

- High-level peer review and editorial services

- Freely accessible online immediately upon publication

- Authors retain the copyright to their work

- Licensing it under a Creative Commons license

- Visibility through different online platforms 